\section{In the Spotlight}

\author{
Maria Rosa Maduro, PhD
}

\section{A Deeper Look into Oocyte Recruitment}

In order to assure the appropriate length of an organism's reproductive lifespan, most of the female oocytes remain in a quiescent state in the ovary for several years. In humans, for example, oocytes can be kept in a dormant state for as long as 50 years and only a limited number of dormant oocytes are progressively recruited into the growing pool. The awakening, development, and differentiation of the female germ cells into mature oocytes able to be fertilized and to lead to a successful pregnancy is dependent on a unique microenvironment, in which the surrounding somatic cells named primordial follicle granulosa cells (pfGCs) are known to play a major role. However, the mechanism by which pfGCs control the activation of primordial follicles and the development of the quiescent oocytes has remained obscured.

Zhang and colleagues have just recently reported new data in the journal Current Biology ${ }^{1}$ that further elucidates the signaling pathways governing the communication between the pfGCs and the oocytes that leads to the recruitment of the last ones. Mechanistic target of rapamycin complex 1 (mTORC1) is a conserved serine/threonine kinase that regulates cell growth and metabolism in response to growth factors and nutrients.

It functions by modulating processes such as protein synthesis, ribosome biogenesis, and autophagy and has been shown to be somehow implicated in pfGCs signaling, as demonstrated by the publication by Li et al in this issue of Reproductive Sciences. ${ }^{2}$

By targeting the somatic pfGCs in mouse models, Zhang et al have now demonstrated that inhibition of mTORC1 signaling in pfGCs prevents the differentiation of pfGCs into granulosa cells, leading to the arrest of the dormant oocytes in their quiescent states and eventually to oocyte death. On the hand, the authors showed that overactivation of mTORC1 signaling in pfGCs accelerates the differentiation of pfGCs into granulosa cells and causes premature activation of all quiescent oocytes and primordial follicles. In addition, Zhang and colleagues evidenced that pfGCs trigger the recruitment of dormant oocytes through KIT ligand (KITL), via the mTORC1-KITL signaling pathway in the somatic cells and the KIT phosphatidylinositol 3-kinase cascade in the oocytes.

In sum, the results presented by Zhang et al provide a relatively complete picture of how mammalian primordial follicles are activated at the molecular level. This knowledge was long sought for as it is essential to develop new clinical approaches to treat certain forms of female infertility.
Reproductive Sciences

2015, Vol. 22(I) 5

(C) The Author(s) 2014

Reprints and permission:

sagepub.com/journalsPermissions.nav

DOI: I0.I|77/|9337|9||456|429

rs.sagepub.com

(S)AGE

\section{Identification of One of the Most Mutated Genes in Endometrial Cancer}

Wingless-related integration site (Wnt) signaling is one of the key oncogenic pathways in multiple cancers, thus targeting this pathway is seen as an attractive therapeutic approach. RNF43, the E3 ubiquitin-protein ligase, is known to negatively regulate the Wnt signaling pathway. Somatic mutations in RNF43 have been associated with heightened sensitivity to compounds that target the Wnt-specific acyltransferase porcupine (PORCN), which is necessary in the processing of Wnt ligand secretion. RNF43 is frequently mutated in several types of cancer, although it had never been before significantly identified in colorectal or endometrial carcinomas.

Unexpectedly, Giannakis et al observed the presence of a large number of nonsilent somatic mutations in RNF43 while performing whole-exome sequencing of colorectal adenocarcinomas and endometrial carcinomas in a sample-control paired study. ${ }^{3}$

Giannakis and colleagues'study, which was recently published in the journal Nature Genetics (Nat Genet advanced online publication, November 2014), reports that RNF43 mutations are present in over $18 \%$ of the colorectal and endometrial cancer samples investigated. The authors demonstrate that truncating mutations of RNF43 are more prevalent in microsatellite unstable tumors and show mutual exclusivity with inactivating APC mutation in colorectal adenocarcinomas.

The results presented by Giannakis et al indicate that RNF43 is one of the most commonly mutated genes in colorectal and endometrial cancers. These findings not only may have a major impact at the clinical level on the fight against cancer but also support the notion that large-scale genomic analysis presents a good tool to identify biologically and clinically relevant mutations in human diseases.

\section{References}

1. Zhang H, Risal S, Gorre N, et al. Somatic cells initiate primordial follicle activation and govern the development of dormant oocytes in mice. Current Biol. 2014;24(21):2501-2508.

2. $\mathrm{Li} \mathrm{L}, \mathrm{Fu} \mathrm{Y}, \mathrm{Xu}$ J, et al. Caloric restriction promotes the reserve of follicle pool in adult female rats by inhibiting the activation of mammalian target of rapamycin signaling. Reprod Sci. 2015;22: 60-67.

3. Giannakis M, Hodis E, Mu XJ, et al. RNF43 is frequently mutated in colorectal and endometrial cancers [published online ahead of print October 26, 2014]. Nat Genet. doi:10.1038/ng.3127. 